\title{
Increased somatic sensations are associated with reduced limb ownership
}

- Kirsten J. McKenzie

- Roger Newport

doi:10.1016/j.jpsychores.2014.11.005

Highlights

We used novel technology to present a purely top-down visual illusion to induce somatosensation.

There was a significant increase in reports of somatosensation during the illusion condition.

Individuals with higher SDQ scores were more susceptible to illusory sensations in all conditions.

There was a strong negative relationship between SDQ score and hand ownership in all conditions.

Those reporting more visually induced somatosensations felt less ownership over their hands.

\section{Abstract}

\section{Objective}

Medically unexplained symptoms (MUS) are increasingly being thought of as resulting from dysfunctional modulation of interoceptive sensory signals by top-down cognitive processes. The current study investigated whether 
individuals with a tendency toward MUS would be more susceptible to visual illusions that suggest tactile sensation on the skin in the absence of any actual somatosensory input.

\section{Method}

Participants viewed real-time-mediated reality video images of their own hand, either un-manipulated or digitally altered to display moving pixelated 'static' effect, the crawling skin illusion. The strength of various physical sensations during each condition were rated on a numeric scale and compared to standard measures of somatoformdissociation (Somatoform Dissociation Questionnaire 20).

\section{Results}

Participants reporting a higher degree of somatoform dissociation were found to be more susceptible to somatic sensations across all conditions. Interestingly, participants who reported more visually induced somatosensory sensations also felt less ownership over their digitally presented hands.

\section{Conclusion}

These findings support the proposed link between MUS and disturbances in body representation, and suggest that an over-reliance on top-down knowledge may interfere with current sensory inputs, contributing to symptom formation and maintenance in susceptible individuals.

Keywords

- Medically unexplained symptoms;

- MIRAGE;

- Illusory sensation;

- Body representation;

- Crawling skin illusion

\section{Introduction}

The interpretation of any incoming sensory information depends upon both the reliability of the signal and the effect of prior experience, so that although our somatic perceptionsseem to reflect reality, bodily events are often 
misjudged or misinterpreted, resulting in distorted bodily experiences. This appears to be the case for individuals experiencing medically unexplained symptoms (MUS), that is, physical symptoms occurring in the apparent absence of physical or psychological pathology [1]. MUS are a trait-like phenomenon distributed across both healthy and clinical populations and are thought to account for up to one third of all medically relevant symptoms in primary care [2] with a greater prevalence in the young and middleaged [3]. Traditionally thought to be the result of abnormal interplay between behavioural, cognitive and physiological processes, recent research suggests that rigid top-down processes during the monitoring of body representation may be an important factor in determining susceptibility to MUS [e.g. 1,4,5]. For individuals with MUS-like tendencies, discrepancies between incoming bottom-up sensory information and current top-down knowledge may result in the sensory input being disregarded in favour of pre-existing top-down schemata. We tested this hypothesis directly, through the use of a novel perceptual illusion - the 'crawling skin' illusion.

Somatic preoccupation or perceptual aberration has been found to be associated with the strength of induced sensations in a number of sensory illusions [4], [5], [6] and [7], including the rubber hand illusion (RHI [8]). While it has been argued that resistance to the $\mathrm{RHI}$ in those susceptible to MUS reflects a trait-like dependence upon top-down information [1] and [5], it is widely accepted that the $\mathrm{RHI}$ involves a combination of both top-down and bottom-up processes (e.g. [9]). In order to investigate the links between MUS and purely top-down influences on bodily perception, we created a new illusion that influences somatosensation solely through visual manipulation. The 'crawling skin' illusion generates a visual effect on the participant's hand that simulates movement on the skin but, unlike the $\mathrm{RHI}$, involves no tactile or somatic sensory input.

If individuals with a higher tendency to experience somatic MUS symptoms exhibit an increased reliance upon top-down processes, we hypothesised that this should result in an increase in somatic sensations reported, in comparison to participants scoring lower on measures of MUS, particularly during the illusion. In keeping with previously reported studies [e.g. 5], we also predicted that participants with higher MUS scores would report lower feelings of limb ownership.

\section{Methods}

\section{Participants}


Twenty-two naïve right-handed participants reporting normal or corrected-tonormal vision and no sensory deficits took part ( 7 males, aged 18 to 23 years [mean $\pm S D=19.82 \pm 1.44$ years]). Participants gave informed consent prior to taking part and procedures were approved by the local ethics committee, in accordance with the 1964 Declaration of Helsinki.

\section{Materials}

Experimental procedures were conducted using a Newport-MIRAGEmediated reality system (University of Nottingham), whereby participants were able to view real-time video images of their own moving hand (delay $<10 \mathrm{~ms}$ ) presented from the same position and from the same visual perspective as if viewing the real limb directly [10]. Experimental conditions showed a real-time image of the hand that was unmanipulated (veridical), a darkened control condition that matched the luminance level inherent in the illusion version (darkened) and a randomly changing selection of pixels on the hand replaced by black pixels (static) (Fig. 1).

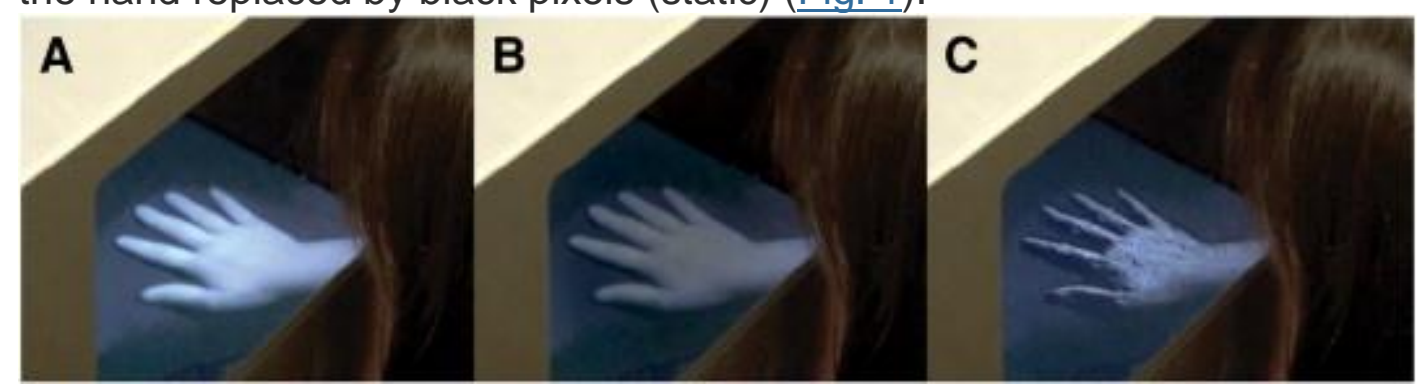

Fig. 1.

MIRAGE visual manipulations: $(A)$ veridical, $(B)$ darkened and (C) static.

\section{Design and procedure}

\section{Questionnaires}

Self-reported MUS experience was measured using the Somatoform Dissociation Questionnaire (SDQ-20 [11]), which asks participants to rate their experience of 20 physical symptoms in the past year. Symptoms that had been medically explained were excluded. Participants also completed the Health Anxiety Inventory (HAI [12]) as health anxiety has been reported to be more prevalent in MUS populations [e.g. 13] and overlaps at least partially with the experience of these symptoms [14]. Experimenters remained blind to both SDQ-20 and HAI Scores during data collection. 
Participants viewed their stationary hand for 2 min in each of three visual conditions [veridical/darkened/static], counterbalanced across participants. After $2 \mathrm{~min}$, participants responded to a series of statements relating to their somatosensory experience and degree of hand ownership, including statements concerned with physical sensations such as 'I can feel a tingling sensation in my hand' and rated each on a scale of scale of 0 ('I strongly agree) to 10 ('I strongly agree'). Following each set of statements, they removed their hand from MIRAGE for 2 min and were encouraged to touch and move it to 'reset' sensation. These breaks were also used for informal discussions of the preceding condition.

\section{Results}

To examine interoceptive somatic sensations reported during each condition, a 'somatosensation score' was calculated from the mean of responses to questions relating to tingling, pins-and-needles touch, itching and throbbing and pleasant/unpleasant sensations. A one-way ANOVA revealed a significant main effect of visual condition $\left(F_{(1.456,}\right.$ 29.13) $=8.799, p=.003$ ); with no significant difference between somatosensory scores for the veridical and darkened conditions $\left(t_{(21)}=-0.184, p=.856\right)$ but much higher somatosensory scores in the static condition compared to the veridical $\left(t_{(21)}=-3.652, p=.002\right)$ and darkened $\left(t_{(21)}=-2.968, p=.008\right)$ conditions (Bonferroni corrected $\alpha=.017$ ). During and after the static condition, several participants made spontaneous comments such as '[it felt] tingly'; 'now I'm getting tingly finger tips' and 'it really tickles! [onset of laughter]'.

A partial correlation analysis, controlling for $\mathrm{HAl}$ score, was conducted between somatosensation and SDQ-20 scores to determine the relationship between the tendency to experience visually induced somatosensations and somatoform dissociation more generally. Significant positive correlations were found between SDQ-20 scores and induced somatosensation across all three conditions: veridical $\left(r_{(18)}=.620, p=.004\right)$, darkened $\left(r_{(18)}=.748, p<.001\right)$ and static $\left(r_{(18)}=.622, p<.003\right)$. When we further calculated a difference score between the veridical and the static conditions, however, we did not find a correlation between SDQ-20 score and somatosensory scores $\left(r_{(18)}=.263, p=.249\right)$. Taken together, these results suggest that participants reporting a higher degree of somatoform dissociation on the SDQ-20 also experienced a higher number of somatic sensations, regardless of visual condition. 
Interestingly, when using the same partial correlation analysis, SDQ-20 scores were found to have significant negative relationships with hand ownership across all three conditions: veridical $\left(r_{(18)}=-.697, p=.001\right)$, darkened $\left(r_{(18)}=-.692, p=.001\right)$ and static $\left(r_{(18)}=-.601, p=.005\right)$, indicating that participants with higher reported levels of somatoform dissociation felt less ownership of their digitally presented hands. Most importantly, there were also very strong significant negative correlations between ownership and somatosensation across all three conditions: veridical $\left(r_{(21)}=-.728, p<.001\right)$, darkened $\left(r_{(21)}=-.953, p<.001\right)$ and static $\left(r_{(21)}=-.656, p=.001\right)$, indicating that participants who felt less ownership over their digitally presented hands also reported feeling more visually induced somatosensory sensations.

\section{Discussion}

The crawling skin illusion did indeed increase reports of illusory somatosensation in comparison to control conditions, through the application of a purely visual manipulation. As predicted, participants with higher somatoform dissociation reported more somatosensation across all three visual conditions. This is consistent with evidence that higher SDQ-20 scorers report more touch sensations in response to visual stimuli when no touch is present [4], and in accordance with an over-reliance upon top-down schema when interpreting bottom-up physiological changes within the body, which lies at the heart of MUS [15]. However, once the disproportionate response of the higher SDQ-20 scorers to baseline conditions had been factored out, the crawling skin illusion condition did not produce an SDQdependent increase in somatosensation, as higher scorers also reported more somatosensation in the unmanipulated baseline condition. This suggests that these individuals may have an elevated sensory baseline in which interoceptive 'noise' is more often misinterpreted as 'signal', regardless of current sensory input. Previous investigations into this area have indicated that such top-down modulation of interoceptive information is due to increased visual attention directed toward the limb[16] and [17]. Indeed, previous research with clinical MUS populations has shown that these individuals are more likely to be hyper-vigilant with respect to bodily symptoms[18], particularly those that are visual in nature. This highlights the importance of including direct-view and non-visual baseline conditions in future illusion experiments.

Higher SDQ-20 scorers also reported lower feelings of ownership over their hands, which is in keeping with the results reported by Miles et al. [5], 
whereby participants with high SDQ-20 scores also reported lower feelings of ownership in the RHI. A novel finding here is that participants with higher SDQ-20 scores experienced reduced ownership over their own limb rather than a proxy limb. This further strengthens the case that those susceptible to MUS may be less in tune with sensory signals emanating from within their own body. In addition, ownership had a strong negative relationship with somatosensation, in that the lower the ownership score, the more participants reported visually induced somatosensation. It is unclear whether reduced ownership causes increased somatic illusions or vice versa, but a similar phenomenon has been observed in patients with Complex Regional Pain Syndrome (CRPS); such individuals experience pain that is far in excess of that expected following recovery from the physiological damage that triggered the CRPS. These patients not only experience a loss of ownership over their affected limb [19] and [20], but pain ratings have also been observed to reduce under conditions in which ownership of the affected limb is increased [20].

This experiment confirms that those reporting higher numbers of MUS are more susceptible to induced somatic sensations. Such distortions are due to an over-reliance upon top-down information when interpreting current sensory input, suggesting that intervention treatments for MUS should focus on improved perception and interpretation of low-level sensory signals, particularly in relation to body representation and ownership.

\section{Conflict of interest}

The authors of this article declare no conflict of interest.

Work on this project by Kirsten J McKenzie was supported by an eScience Fund Grant from the Malaysian Ministry of Science, Technology and Innovation [06-02-12-SF0158]. This funding body had no involvement in study design, collection, analysis and interpretation of data or in the writing of the report nor any decision to submit for publication.

The authors declare that this paper has not previously been published in any form and is not under consideration with another journal.

\section{Acknowledgements}

Work on this project by KJM was supported by an eScience Fund Grant from the Malaysian Ministry of Science, Technology and Innovation [06-0212-SF0158]. This funding body had no further involvement. The authors would also like to thank Conor Jones for his help with data collection. 


\section{References}

1.

○ RJ Brown

- Psychological mechanisms of medically unexplained symptoms: an integrative conceptual model

○ Psychol Bull, 130 (2004), pp. 793-812

2.

- K Kroenke

- Patients presenting with somatic complaints: epidemiology, psychiatric comorbidity and management

- Int J Methods Psychiatr Res, 12 (2003), pp. 34-43

3.

- PH Hilderink, R Collard, JG Rosmalen, RC Oudevoshaar

- Prevalence of somatoform disorders and medically unexplained symptoms in old age populations in comparison with younger age groups: a systematic review

- Ageing Res Rev, 12 (2012), pp. 151-156 http://dx.doi.org/10.1016/i.arr.2012.04.004

4.

- RJ Brown, N Brunt, E Poliakoff, DM Lloyd

- Illusory touch and tactile perception in somatoform dissociators

- J Psychosom Res, 69 (2010), pp. 241248 http://dx.doi.org/10.1016/i.jpsychores.2009.11.010

5.

- E Miles, E Poliakoff, RJ Brown

- Medically unexplained symptom reports are associated with a decreased response to the rubber hand illusion

- J Psychosom Res, 71 (2011), pp. 240-

244 http://dx.doi.org/10.1016/j.jpsychores.2011.04.002

6.

- A Burrack, PB Brugger

- Individual differences in susceptibility to experimentally induced phantom sensations

○ Body Image, 2 (2005), pp. 307-313

7.

- M MacLachlan, D Desmond, O Horgan 
- Psychological correlates of illusory body experiences

- J Rehabil Res Dev, 40 (2003), pp. 59-66

8.

- M Botvinick, J Cohen

- Rubber hands 'feel' touch that eyes see

- Nature, 391 (1998), p. 756

9.

- M Tsakiris

- My body in the brain: a neurocognitive model of body-ownership

- Neuropsychologia, 48 (2010), pp. 703-

712 http://dx.doi.org/10.1016/j.neuropsychologia.2009.09.034

10.

- R Newport, R Pearce, C Preston

- Fake hands in action: embodiment and control of supernumerary limbs

- Exp Brain Res, 204 (2010), pp. 385-395 http://dx.doi.org/10.1007/s00221-009-2104-y

11.

- ER Nijenhuis, P Spinhoven, R Van Dyck, O Van Der Hart, J Vanderlinden

- The development and psychometric characteristics of the Somatoform Dissociation Questionnaire (SDQ-20)

○ J Nerv Ment Dis, 184 (1996), pp. 688-694

12.

- PM Salkovskis, KA Rimes, HM Warwick, DM Clark

- The Health Anxiety Inventory: development and validation of scales for the measurement of health anxiety and hypochondriasis

○ Psychol Med, 32 (2002), pp. 843-853

13.

- J Russo, W Katon, M Sullivan, M Clark, D Buchwald

- Severity of somatization and its relationship to psychiatric disorders and personality

○ Psychosomatics, 35 (1994), pp. 546-556

14.

- H Tryer

○ Tackling Health Anxiety: A CBT Handbook

○ RCPsych Publications, London (2013) 
15.

- K Bogaerts, L Van Eylen, W Li, J Bresseleers, I Van Diest, S De Peuter, et al.

- Distorted symptom perception in patients with medically unexplained symptoms

○ J Abnorm Psychol, 119 (2010), pp. 226-234 http://dx.doi.org/10.1037/a0017780

16.

- L Mirams, E Poliakoff, RJ Brown, DM Lloyd

- Vision of the body increases interference on the somatic signal detection task

- Exp Brain Res, 202 (2010), pp. 787-794 http://dx.doi.org/10.1007/s00221-010-2185-7

17.

- M Taylor-Clarke, S Kennett, P Haggard

$\circ$ Persistence of visual-tactile enhancement in humans

○ Neurosci Lett, 354 (2004), pp. 22-25

18.

○ RJ Brown

○ Medically unexplained symptoms: a new model

○ Psychiatry, 5 (2006), pp. 43-47 http://dx.doi.org/10.1383/psyt.2006.5.2.43

19.

- JS Lewis, P Kersten, CS McCabe, KM McPherson, DR Blake

- Body perception disturbance: a contribution to pain in complex regional pain syndrome (CRPS)

- Pain, 133 (2007), pp. 111-119

20.

- L Moseley, T Parsons, C Spence

- Visual distortion of a limb modulates the pain and swelling evoked by movement

- Curr Biol, 18 (2008), pp. R1047-R1048 http://dx.doi.org/10.1016/i.cub.2008.09.031

Corresponding author. Tel.: + 64 226734575; fax: + 60389248018 .

Copyright (@ 2014 Elsevier Inc. All rights reserved 